\title{
Extraordinary case presentations in pediatric pituitary adenoma: report of 6 cases
}

\author{
Jenna Meyer, MS, Avital Perry, MD, Soliman Oushy, MD, Christopher S. Graffeo, MD, MS, \\ Lucas P. Carlstrom, MD, PhD, and Fredric B. Meyer, MD \\ Department of Neurologic Surgery, Mayo Clinic, Rochester, Minnesota
}

Pediatric pituitary adenomas (PPAs) are rare neoplasms with a propensity for unusual presentations and an aggressive clinical course. Here, the authors describe 6 highly atypical PPAs to highlight this tendency and discuss unexpected management challenges.

A 14-year-old girl presented with acute hemiparesis and aphasia. MRI revealed a pituitary macroadenoma causing internal carotid artery invasion/obliteration without acute apoplexy, which was treated via emergent transsphenoidal resection (TSR). Another 14-year-old girl developed precocious galactorrhea due to macroprolactinoma, which was medically managed. Several years later, she re-presented with acute, severe, bitemporal hemianopia during her third trimester of pregnancy, requiring emergent induction of labor followed by TSR. A 13-year-old boy was incidentally diagnosed with a prolactinoma after routine orthodontic radiographs captured a subtly abnormal sella. An 18-year-old male self-diagnosed pituitary gigantism through a school report on pituitary disease. A 17-year-old boy was diagnosed with Cushing disease by his basketball coach, a former endocrinologist. A 12-year-old girl with growth arrest and weight gain was diagnosed with Cushing disease, which was initially treated via TSR but subsequently recurred and ultimately required 12 operations, 5 radiation treatments involving 3 modalities, bilateral adrenalectomy, and chemotherapy. Despite these efforts, she ultimately died from pituitary carcinoma.

https://thejns.org/doi/abs/10.3171/2019.7.PEDS1950

KEYWORDS pediatric; adenoma; prolactinoma; extraordinary; pituitary surgery

$\mathrm{P}$ EDIATRIC pituitary adenomas (PPAs) are rare neoplasms that account for $2 \%$ of all pediatric brain tumors. ${ }^{32}$ Typical clinical presentation depends on the extent of mass effect and, for secreting tumors, which trophic hormones are dysregulated. In addition to headaches, children frequently come to medical attention because of visual disturbances, growth failure, or abnormal pubertal onset. ${ }^{15,18}$ Interestingly, PPA appears to have a penchant for unusual presentations, atypical symptomatologies, or extremes of severity. When these tendencies are combined with the lesion's relative rarity, frequent misdiagnosis occurs, as do a host of uncommon management challenges. We report several examples of particularly striking PPA presentations to illustrate the potential for the unexpected in this rare disease, as well as to demonstrate several generalizable lessons in the management of PPA.

A prospectively maintained neurosurgical database at a large tertiary care academic referral center was retrospectively queried for cases of pituitary adenomas in patients 18 years old or younger during the study period, 1995-2017. Secondary chart review identified 6 cases with particularly striking presentations and/or extraordinary challenges in both diagnosis and treatment. This study was approved by our institutional review board, including a waiver of consent given the minimal-risk study design.

\section{Case Reports}

\section{Case 1: Pituitary Adenoma Presenting as Acute Stroke}

A 14-year-old girl presented to an outside facility with a 24-hour history of aphasia and right paresis concerning for an acute infarct. She reported progressive headaches, nausea, and photophobia for the 2 months prior to presentation. Laboratory testing revealed a prolactin of $3971 \mathrm{ng} /$ $\mathrm{ml}$ and thyroid-stimulating hormone of $0.7 \mathrm{mIU} / \mathrm{L}$. MRI and angiography revealed an invasive $33-\mathrm{mm}$ sellar-suprasellar mass with significant compression of the optic chiasm and the left internal carotid artery (ICA), as well

ABBREVIATIONS ACTH = adrenocorticotropic hormone; $\mathrm{CD}=$ Cushing disease; $\mathrm{GH}=$ growth hormone; $\mathrm{GHRH}=\mathrm{GH}$-releasing hormone; ICA = internal carotid artery; IGF-1 = insulin-like growth factor 1; PG = pituitary gigantism; PPA = pediatric pituitary adenoma; $\mathrm{SRS}=$ stereotactic radiosurgery; TSR = transsphenoidal resection. SUBMITTED March 4, 2019. ACCEPTED July 30, 2019.

INCLUDE WHEN CITING Published online October 11, 2019; DOI: 10.3171/2019.7.PEDS1950. 

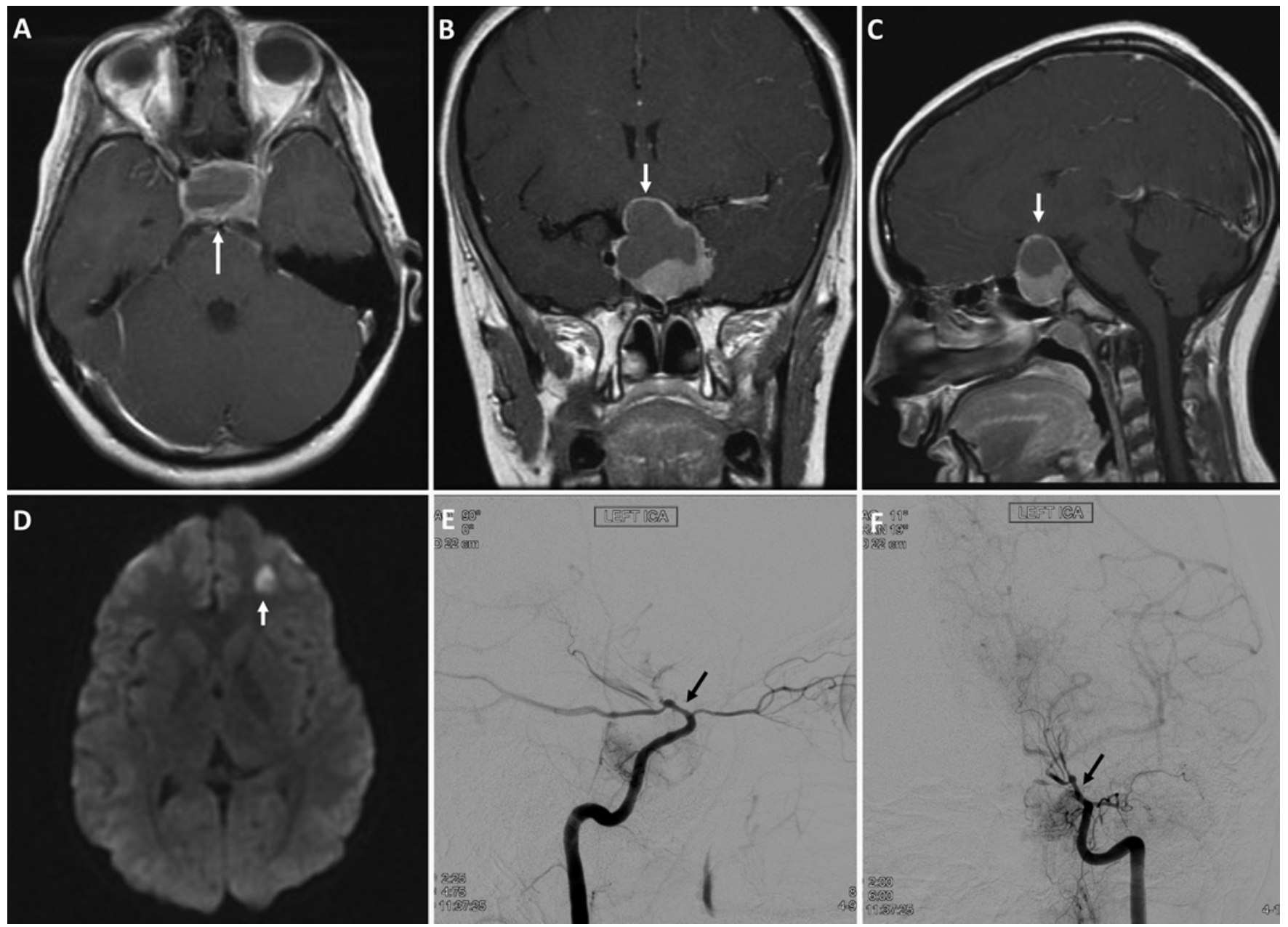

FIG. 1. Case 1. Preoperative contrast-enhanced axial (A), coronal (B), and sagittal (C) T1-weighted MRI demonstrated a large, heterogeneously enhancing sellar mass with suprasellar extension and left ICA encasement (white arrows). Axial diffusion-weighted imaging (D) identified a left posterior frontal lobe infarct (white arrow). Stenosis of the left ICA distal to the petrous segment (black arrows) was confirmed on lateral (E) and anteroposterior (F) cerebral angiography.

as a resulting infarct within the left frontal lobe (Fig. 1). She was taken to the operating room emergently for subtotal surgical decompression of a histopathologically confirmed prolactinoma via a transnasal endoscope-assisted approach and transsphenoidal resection (TSR). Postoperatively, she required chronic cabergoline pharmacotherapy, as well as supplemental thyroid hormone, growth hormone (GH), adrenocorticotropic hormone (ACTH), and gonadotropins. Total duration of follow-up was 58 months. During her most recent follow-up, she was able to ambulate independently with mild residual hemibody weakness and minor expressive aphasia, serial MRI was negative for tumor recurrence, and she was successfully weaned off all hormone replacement with the exception of cabergoline therapy.

\section{Case 2: Pregnancy-Induced Rapid Progression Requiring} Emergent Induction

This case represents an exceedingly rare intersection of conditions that, while uncommon independently, have never been reported in concert. A 14-year-old girl with galactorrhea was diagnosed with a pituitary prolactinoma, which was managed medically with cabergoline (Fig. 2A). Her tumor remained stable in size without abnormalities on formal visual field testing over 3 years of medical management. At the age of 17 years, the patient became pregnant, and the cabergoline was discontinued without neurological follow-up. During her third trimester, she developed isolated progressive bitemporal hemianopia concerning for progression of her known prolactinoma. At 36 weeks' gestation, she developed marked subacute visual loss confirmed on visual field testing (Fig. 2C), prompting transfer to our facility for obstetric and neurosurgical care. MRI showed clear tumor expansion with compression of the optic chiasm without evidence of apoplexy (Fig. 2B), and cabergoline was initiated. She progressed to neartotal visual loss over several days despite high-dose bromocriptine therapy. Labor was induced, followed shortly thereafter by TSR on postpartum day 1 . She had an uncomplicated recovery with normalization of visual func- 

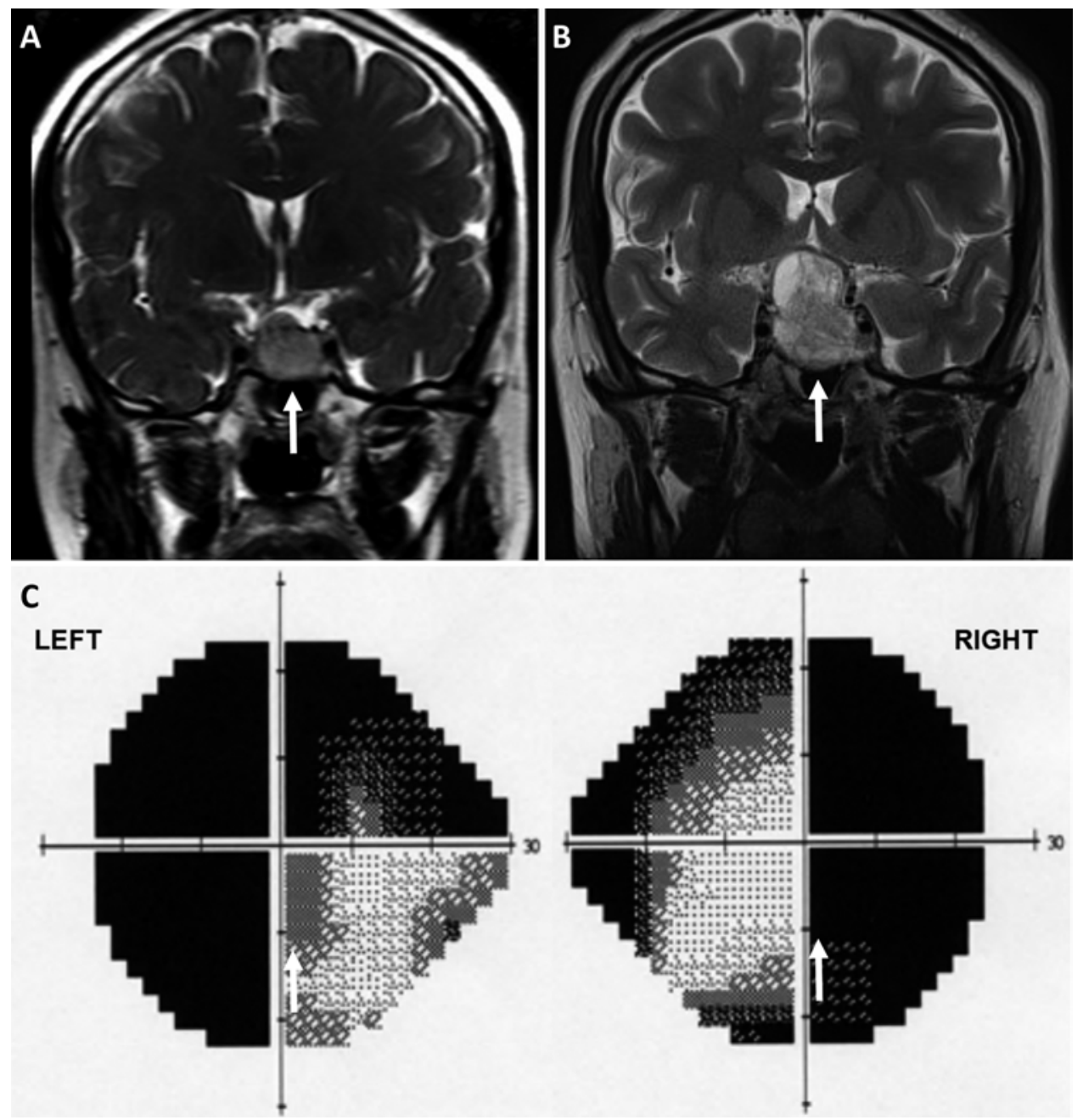

FIG. 2. Case 2. Serial coronal T2-weighted MRI studies demonstrated marked interval enlargement of the pituitary tumor from initial diagnosis $(\mathrm{A})$ to recurrence during pregnancy $(\mathrm{B})$. Contrast-enhanced imaging was not obtained because of gravid status. Formal visual field testing $(\mathbf{C})$ confirmed bitemporal hemianopia consistent with compression of the optic chiasm.

tion, as well as a pronounced decrease in serum prolactin from 2629 to $189 \mathrm{ng} / \mathrm{ml}$. She was discharged on bromocriptine therapy. Unfortunately, she was lost to follow-up.

\section{Case 3: Serendipitous Prolactinoma Discovery}

An asymptomatic 13-year-old boy underwent maxillofacial radiographs as part of a routine orthodontic assessment. Subtle sellar abnormalities were noted, leading to subsequent brain MRI, which demonstrated a cystic sellar mass with extension into the sphenoid sinus and suprasellar cistern. Unfortunately, preoperative images were not available for review. Formal visual field testing documented mild bitemporal hemianopia. Endocrinological assessment confirmed a serum prolactin level of $793 \mathrm{ng} / \mathrm{ml}$. The patient underwent endoscopic TSR without complications. Histopathological analysis demonstrated an adenoma with predominant prolactin- and GH-positive regions interspersed with mixed chronicity apoplectic changes. He developed no new endocrine deficits, and total resolution of the visual field deficits occurred postoperatively. He required chronic bromocriptine treatment for persistent hyperprolactinemia, as well as hormonal replacement therapy for secondary hypogonadism. The total duration of follow-up was 186 months. He was asymptomatic at his most recent follow-up visit in the clinic.

\section{Case 4: Self-Diagnosed Pituitary Gigantism}

While completing a school assignment on acromegaly, an 18-year-old male noted a number of familiar features, including rapid peripubertal growth to a final height of $217 \mathrm{~cm}$, hyperhidrosis, headaches, and voice changes. He had no personal or family history of pituitary disease. Formal visual field testing was unremarkable. Laboratory analysis was remarkable for a baseline insulin-like growth factor 1 (IGF-1) of $845 \mathrm{ng} / \mathrm{ml}$, subthreshold GH-releasing hormone (GHRH) level, and positive GH suppression on glucose challenge. MRI of the brain demonstrated a 10$\mathrm{mm}$, partially cystic, midline pituitary mass for which he 

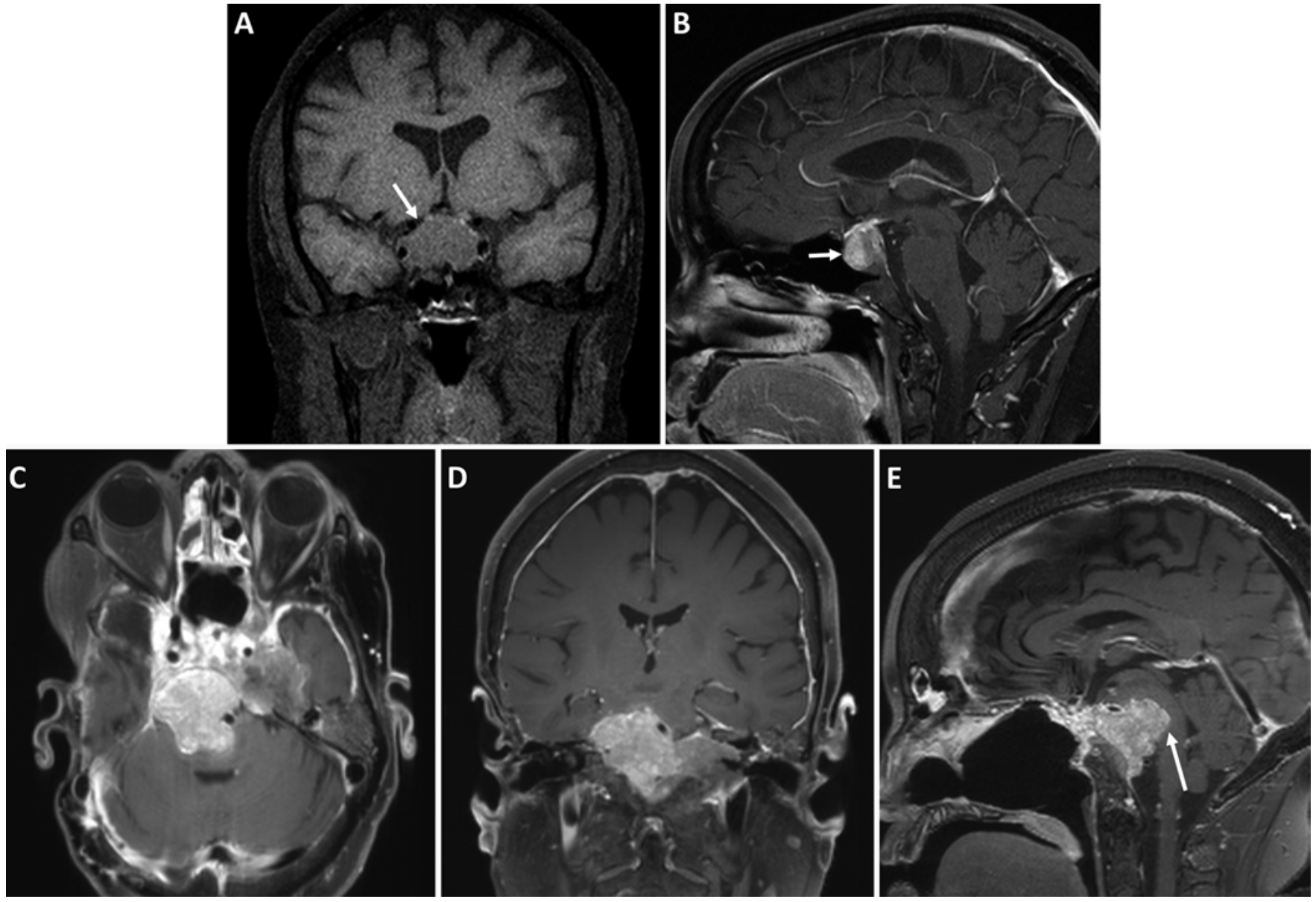

FIG. 3. Case 6. Preoperative contrast-enhanced coronal (A) and sagittal (B) T1-weighted MRI at the time of diagnosis demonstrated a sellar mass (white arrows) with variable gadolinium uptake. Interval tumor progression was dramatically observed in the axial (C), coronal (D), and sagittal (E) planes, with clear brainstem compression (white arrow) best appreciated on sagittal sequences.

underwent subtotal resection via endoscopic TSR. Postoperatively, IGF-1 was persistently elevated, and the patient underwent repeat TSR 5 months later without neurological deficits postoperatively. The total duration of follow-up was 7 months. IGF-1 levels remained stable with preservation of endocrine function following a 2-month period of corticosteroid replacement.

\section{Case 5: Unusual Referral for Cushing Syndrome}

A 17-year-old male was referred by his basketball coach, a former endocrinologist, who noted several body habitus changes including a buffalo hump, weight gain, and abdominal striae, most consistent with classic Cushing syndrome. Initial consultation confirmed an elevated serum ACTH concentration, which was suppressed on administration of high-dose dexamethasone. MRI of the brain revealed a nonenhancing nodule centered within the right anterior pituitary gland. The patient underwent endoscopic TSR, resulting in durable tumor control and stable neurological function. Postoperatively, he required pharmacological supplementation for hypocortisolism. Final histopathology confirmed an ACTH-positive adenoma.
The patient elected to follow-up locally after 1 month of care at our institution.

Case 6: Remarkably Aggressive, Treatment Refractory, Atypical Cushing Disease

Our most complex case was largely cared for at outside institutions. This female was found to have an ACTH-secreting microadenoma at age 12 years in the setting of arrested growth and weight gain (Fig. 3A and B). She initially underwent gross-total resection via TSR. Postoperatively, she required GH supplementation. Several years later, she presented with visual loss and Cushingoid-like symptoms, indicating a large biochemical recurrence, which was treated with repeat TSR and adjuvant stereotactic radiosurgery (SRS). Two years later, she relapsed during pregnancy and underwent a third resection, external beam radiotherapy, and a 6-month course of ketoconazole, after which she returned to her asymptomatic baseline. Four years later, her symptoms recurred, and imaging showed an expansive tumor with significant ICA encasement and suprasellar disease, for which she underwent a craniotomy complicated by sixth cranial nerve palsy, CSF leakage, 
meningitis, and hydrocephalus requiring placement of a ventriculoperitoneal shunt. Persistent disease prompted a fourth TSR and fractionated linear accelerator (LINAC)based SRS, which was followed by a 1-year symptom-free interval before another recurrence prompted a second craniotomy and several rounds of temozolomide chemotherapy, with intermittent severe waxing and waning of symptoms throughout.

At this point in her history, the patient was referred to our institution, where she initially underwent bilateral adrenalectomy for severe, refractory Cushing disease (CD), followed by four rounds of adjuvant paclitaxel and cisplatin, which failed to arrest tumor growth. Octreotide was subsequently trialed and similarly failed, and the patient underwent fractionated SRS. Remarkably, tumor growth persisted (Fig. 3C-E), and the patient developed symptomatic brainstem compression with left leg weakness, prompting an attempted resection via anterior petrosectomy. Unfortunately, only a very limited debulking could be achieved because of extreme fibrosis. Histopathological studies were consistent with a recurrent atypical corticotroph-type adenoma with positive staining for ACTH, low overall p53 labeling with strong staining in cell subsets, and an elevated Ki-67 labeling. Cyclophosphamide, vincristine, and dacarbazine were initiated but aborted during the third cycle because of neutropenic sepsis. Although she recovered medically, the patient suffered a left hypothalamic infarct, thought to be attributable to hypotension in the setting of radiation-induced vasculopathy. Bevacizumab was initiated, and a posterior petrosectomy was eventually attempted, through which a much more extensive debulking was completed. Repeat pathological analysis showed 8 mitoses per $10 \mathrm{hpf}$, an increase from the previous resection, and positive staining for chromogranin; BRAF V600E was negative.

Postoperatively, the patient's strength improved moderately, allowing her to ambulate and eventually discharge to acute inpatient rehabilitation. At the last follow-up 42 weeks after transfer to our facility, she was wheelchair bound with multiple cranial nerve palsies secondary to tumor progression-despite chemotherapy. The patient decided to pursue palliative care and ultimately died of her disease 26 years after diagnosis.

\section{Discussion}

This report highlights a subset of 6 extreme PPA outliers to synergistically illustrate an overarching point regarding our experience with the management of PPA: it is a clinically expansive and potentially dangerous disease capable of dramatic, delayed recurrences possibly requiring the full breadth of aggressive and creative treatment options. As we have described previously, the spectrum of typical pediatric adenoma patients is diverse at baseline, yet the most common symptoms still fall within the range of conceivable pituitary pathophysiology-such as headache, obesity/weight gain, visual disturbance, acne, amenorrhea, hirsutism, or moon facies. ${ }^{31}$ Against this backdrop, the current series emphasizes the increased propensity for truly extraordinary clinical presentations within this particularly vulnerable patient population.

\section{Internal Carotid Artery Compression Secondary to Pituitary Adenoma}

We report the first case of ICA infarction in a pediatric patient attributable to subacute critical stenosis from pituitary tumor invasion and compression, as opposed to acute apoplexy. Invasive macroadenomas have been reported to cause symptomatic cranial nerve deficits in the setting of pituitary apoplexy or extreme mass effect, most commonly involving cranial nerves III, IV, or VI. ${ }^{17}$ Similarly, pituitary apoplexy has been reported to cause symptomatic ICA obstruction via either direct compression or vasospasm secondary to apoplectic subarachnoid hemorrhage. , $, 34,43$ Compression of the ICA by an invasive macroadenoma in the absence of apoplexy is very rare and often asymptomatic, especially in pediatric patients. ${ }^{27}$ In our patient, chronic intratumoral blood products were noted, but they were scattered, scant, and inconsistent in terms of time course to explain the acute presentation. In lieu of acute apoplexy, we hold that perivascular spread resulting in direct adenoma invasion of the supraclinoid ICA led to severe stenosis, occlusion, and, ultimately, infarction.

Four prior cases of symptomatic ICA compression in the absence of pituitary apoplexy in any age group have been reported, occurring at an average age of 56 years. $2,7,34,39$ Alentorn et al. reported a case of recurrent pituitary tumor presenting with right paresis and aphasia. Imaging demonstrated a large pituitary adenoma recurrence with clear occlusion of the encircled ICA; however, the primary tumor had been treated with subtotal resection followed by external beam radiation at 45 Gy approximately 32 years earlier, raising the possibility of radiation-induced arteriopathy as a contributor to the eventual infarct. ${ }^{2}$ Cavalcanti and de Castro Júnior reported ICA stenosis in a newly diagnosed nonfunctioning pituitary adenoma, although in their case the finding may have been incidental, as the more pronounced vascular disease was ipsilateral to the hemiparesis. ${ }^{7}$ Rey-Dios et al. reported a case of left clinoid ICA stenosis caused by an enlarging pituitary adenoma, which presented subacutely with transient episodes of left hemispheric ischemia over several months, accompanied by bitemporal hemianopia. ${ }^{34}$ The patient underwent endonasal transsphenoidal tumor resection, which restored ICA flow and resulted in the resolution of his symptoms. Spallone reported a recurrence of an eosinophilic adenoma 19 months after primary resection, subsequently resulting in an altered mental status and dense left hemiplegia attributable to a right supraclinoid ICA occlusion; because of the severity of the injury, no further treatment was pursued at the time. ${ }^{39}$

\section{Pituitary Prolactinoma in Pregnancy}

Progression of prolactinomas during pregnancy has been reported in up to $32 \%$ of cases. ${ }^{13}$ Pathophysiologically, elevated serum estrogen associated with the normal physiological changes of a healthy pregnancy simultaneously acts on estrogen receptors expressed by prolactinoma cells, resulting in marked tumor enlargement. . $^{3,26}$ Correspondingly, a pregnancy test is strongly advised in women of reproductive age who present with a rapid progression of symptoms-particularly prior to initiating 
a potentially harmful treatment-or in a younger patient who is at highest risk of unintended conception. ${ }^{38}$

Management of prolactinomas during pregnancy in pediatric patients is poorly studied, and current practices are derived from the adult literature; but even in that population, meaningful guidelines regarding timing and prioritization of neurosurgical versus obstetrical interventions are scarce. Dopamine agonist therapy (e.g., bromocriptine or cabergoline) is the first-line standard of care in prolactinoma; however, absent invasive macroprolactinomas, current Endocrine Society practice guidelines recommend discontinuation of dopamine agonist therapy in pregnant patients given the lack of adequate safety data. ${ }^{24}$ In parallel, resection is reserved for medically refractory tumors and cases in which dopamine agonists are poorly tolerated. Under ideal circumstances, if TSR is required during gestation, the second trimester is strongly preferred; alternatively, an early induction may warrant consideration if pregnancy and disease are both quite advanced..$^{10,26}$ Radiotherapy and SRS may also be considered in particularly refractory cases but are categorically avoided in pregnancy.

\section{GH-Secreting Pituitary Adenoma: Gigantism and Acromegaly}

GH excess in pediatric patients without fused epiphyseal growth plates typically results in extremely tall adults, a condition known as "gigantism." "Acromegaly" refers to GH excess in adults with associated sequelae; stature changes are typically absent given the onset of disease after growth cessation. Pituitary gigantism (PG) is a rare disease, accounting for $0.6 \%$ of all cases in children and adolescents with PPA, with a marked male predominance., ${ }^{1,37}$ The majority of PG cases have no known underlying genetic etiology; however, gigantism is a recognized feature of some genetic syndromes including McCune-Albright syndrome and multiple endocrine neoplasia type $1 .{ }^{21,44}$

Patients with PG typically present with dramatically accelerated linear growth, typically without concomitant weight gain-although an increase in both parameters can be noted in a minority of patients. PG is the most likely PPA to present in infancy and early childhood, most frequently with progressive macrocephaly. ${ }^{5}$ Other common features more characteristic of adolescent presentation include hyperhidrosis, frontal bossing, prognathism, amenorrhea, and bony enlargement of the distal extremeties. ${ }^{35}$ IGF-1 is the preferred parameter for both screening and follow-up given that it is overarchingly dependent on endogenous GH secretion, yet not subject to the same wide diurnal variations, thus making it a more reliable metric. For most patients, elevated age-adjusted IGF-1 is confirmed with a high-dose oral glucose challenge test, which will fail to elicit normal GH suppression in patients harboring secretory tumors. In equivocal or ectopic cases, serum GHRH may be helpful in ruling out ectopic GHRH secretion as the underlying etiology. As with most pituitary adenomas, GH-secreting tumors have no characteristic imaging features, and unlike prolactinomas (which skew large) and ACTH-secreting adenomas (which skew small), their size may be highly variable. ${ }^{20,46}$ Provided adequate sphenoid pneumatization, TSR is the treatment of choice in the pediatric population; transcranial resection is an alternative in very young children, while radiotherapy, SRS, and pharmacological suppression with octreotide or bromocriptine is typically reserved for recurrent or refractory disease or lesions that are not amenable to surgery.

\section{Adrenocorticotropic-Secreting Pituitary Adenomas in Pediatric Patients}

As with prolactinomas, ACTH-secreting tumors occur with high frequency in pediatric patients, a sharp contrast to the adult population, in which nonfunctioning tumors dominate. ${ }^{25,29}$ Pediatric CD typically presents during the prepubertal or pubertal period with unexpected growth patterns or an abnormal onset of puberty, with a female/ male predominance of 3:1.25 Thus, symptoms of ACTHsecreting adenomas are particularly insidious in children and are frequently overlooked or misdiagnosed until the disease is quite advanced, making definitive treatment challenging and recurrence likely. This is further complicated not only by the fact that pediatric ACTH-secreting adenomas recur in up to $27 \%$ of patients, almost doubling the 14\%-17\% incidence observed in other secreting PPAs, but also by the fact that patients diagnosed in childhood will have a much more protracted natural history, exponentially increasing the risk of delayed recurrence or progression as well as the general consensus that pediatric $\mathrm{CD}$ is among the most aggressive subgroups of pituitary disease. . $^{8,19,25,30}$

The patient in case 6 illustrates an extreme example of delayed recurrence following an initially routine treatment course. Although not unique to pediatric patients, comparable delays of 10 years or more have been more frequently reported in the childhood CD population, including recurrence at up to 13 years with interval resolution of symptoms ${ }^{28,29}$ Recurrence in the setting of prolonged remission has been hypothesized to be the result of residual microscopic adenoma regrowth, versus de novo adenoma formation in a presumably susceptible patient. ${ }^{28,29}$

Attempts to characterize factors predictive of tumor recurrence have focused on perioperative hormone levels. Mindermann and Wilson conducted a retrospective PPA analysis, in which they concluded that recurrence of ACTH-secreting adenomas, unlike prolactinomas and GH-releasing adenomas, is not correlated with pre-, intra-, or postoperative ACTH levels. ${ }^{25}$ However, postoperative normalization of serum cortisol, ideally tested at 8:00 AM on the first postoperative day, is highly predictive of biochemical CD remission. ${ }^{8,11,36}$ Batista et al. also observed that ovine corticotropin-releasing hormone stimulation testing was a reliable predictor of recurrence, whereas urinary free cortisol values were not clinically useful. ${ }^{4}$

As with other nonprolactinoma PPAs, the treatment of choice for pediatric CD is TSR given adequate sphenoid pneumatization to provide an appropriate operative corridor. Indeed, given the ongoing evolution of expanded endonasal techniques, an increasingly large fraction of sellar pathologies can be treated via the endonasal corridoreven among very young patients without robust pneumatization of the sphenoid. On rare occasions, in which nasal anatomy is particularly limiting, a sublabial transsphenoidal approach may warrant consideration; for very large, invasive tumors, an open craniotomy may be considered. ${ }^{9}$ 
Unique to $\mathrm{CD}$ is the significant differential between lesion size and extent of symptoms, rendering surgery challenging and risky in terms of damage to the normal gland and the case prone to persistent symptoms due to even microscopic residuum. ${ }^{23,33,40-42,45}$ Even in the most experienced hands achieving gross-total resection after careful and thorough examination of the sella, a biochemical cure is frequently not achieved..$^{12,14}$ Treatment options for $\mathrm{CD}$ after failed TSR include repeated surgery, bilateral adrenalectomy, stereotactic radiotherapy, and medical therapy-each of which is subject to a host of limitations. ${ }^{16,22}$ Even after several successful treatments, pediatric $\mathrm{CD}$ has the capacity for incredible refractoriness to both medical and surgical interventions, as evidenced by our patient in case 6 , who died from disease after a decades-long battle in which all imaginable neurosurgical resources were exhausted.

\section{Conclusions}

Management of PPA is characterized by the unexpected, the exceptional, and the challenging - whether in making the diagnosis, managing the disease, or definitively treating a quixotic tumor. Our series represents a selection of extreme cases from the wide swath of interesting and diverse clinical circumstances that can be encountered in PPA, including the first report of a pediatric patient presenting with an ICA infarct attributable to progression of subacute critical stenosis in the context of vascular adenoma invasion, in lieu of acute apoplexy. Ultimately, PPAs are rare, protean tumors that demand the highest level of vigilance and clinical care. Given the minimal, anecdotal evidence specifically pertinent to PPA, we recommend above all that each patient be managed by a multidisciplinary care team with expertise in both pituitary and pediatric disease in order to optimize an individualized and comprehensive approach to treatment.

\section{References}

1. Abe T, Tara LA, Lüdecke DK: Growth hormone-secreting pituitary adenomas in childhood and adolescence: features and results of transnasal surgery. Neurosurgery 45:1-10, 1999

2. Alentorn A, Bruna J, Acebes JJ, Velasco R: Stroke and carotid occlusion by giant non-hemorrhagic pituitary adenoma. Acta Neurochir (Wien) 153:2457-2459, 2011

3. Almalki MH, Alzahrani S, Alshahrani F, Alsherbeni S, Almoharib O, Aljohani N, et al: Managing prolactinomas during pregnancy. Front Endocrinol (Lausanne) 6:85, 2015

4. Batista DL, Oldfield EH, Keil MF, Stratakis CA: Postoperative testing to predict recurrent Cushing disease in children. J Clin Endocrinol Metab 94:2757-2765, 2009

5. Blumberg DL, Sklar CA, David R, Rothenberg S, Bell J: Acromegaly in an infant. Pediatrics 83:998-1002, 1989

6. Cardoso ER, Peterson EW: Pituitary apoplexy and vasospasm. Surg Neurol 20:391-395, 1983

7. Cavalcanti CE, de Castro Júnior AN: [Non-hemorrhagic pituitary macroadenoma producing reversible internal carotid artery occlusion: case report.] Arq Neuropsiquiatr 55 (3B):659-660, 1997 (Portuguese)

8. Chen JC, Amar AP, Choi S, Singer P, Couldwell WT, Weiss $\mathrm{MH}$ : Transsphenoidal microsurgical treatment of Cushing disease: postoperative assessment of surgical efficacy by application of an overnight low-dose dexamethasone suppression test. J Neurosurg 98:967-973, 2003

9. Chivukula S, Koutourousiou M, Snyderman CH, Fernandez-
Miranda JC, Gardner PA, Tyler-Kabara EC: Endoscopic endonasal skull base surgery in the pediatric population. $\mathbf{J}$ Neurosurg Pediatr 11:227-241, 2013

10. Chrisoulidou A, Boudina M, Karavitaki N, Bili E, Wass J: Pituitary disorders in pregnancy. Hormones (Athens) 14:7080,2015

11. Esposito F, Dusick JR, Cohan P, Moftakhar P, McArthur D, Wang C, et al: Clinical review: Early morning cortisol levels as a predictor of remission after transsphenoidal surgery for Cushing's disease. J Clin Endocrinol Metab 91:7-13, 2006

12. Friedman RB, Oldfield EH, Nieman LK, Chrousos GP, Doppman JL, Cutler GB Jr, et al: Repeat transsphenoidal surgery for Cushing's disease. J Neurosurg 71:520-527, 1989

13. Gillam MP, Molitch ME, Lombardi G, Colao A: Advances in the treatment of prolactinomas. Endocr Rev 27:485-534, 2006

14. Joshi SM, Hewitt RJ, Storr HL, Rezajooi K, Ellamushi H, Grossman AB, et al: Cushing's disease in children and adolescents: 20 years of experience in a single neurosurgical center. Neurosurgery 57:281-285, 2005

15. Kane LA, Leinung MC, Scheithauer BW, Bergstralh EJ, Laws ER Jr, Groover RV, et al: Pituitary adenomas in childhood and adolescence. J Clin Endocrinol Metab 79:1135-1140, 1994

16. Kelly DF: Transsphenoidal surgery for Cushing's disease: a review of success rates, remission predictors, management of failed surgery, and Nelson's Syndrome. Neurosurg Focus 23(3):E5, 2007

17. Kim SH, Lee KC, Kim SH: Cranial nerve palsies accompanying pituitary tumour. J Clin Neurosci 14:1158-1162, 2007

18. Lafferty AR, Chrousos GP: Pituitary tumors in children and adolescents. J Clin Endocrinol Metab 84:4317-4323, 1999

19. Leinung MC, Kane LA, Scheithauer BW, Carpenter PC, Laws ER Jr, Zimmerman D: Long term follow-up of transsphenoidal surgery for the treatment of Cushing's disease in childhood. J Clin Endocrinol Metab 80:2475-2479, 1995

20. Lu PW, Silink M, Johnston I, Cowell CT, Jimenez M: Pituitary gigantism. Arch Dis Child 67:1039-1041, 1992

21. Marx S, Spiegel AM, Skarulis MC, Doppman JL, Collins FS, Liotta LA: Multiple endocrine neoplasia type 1: clinical and genetic topics. Ann Intern Med 129:484-494, 1998

22. McArthur RG, Cloutier MD, Hayles AB, Sprague RG: Cushing's disease in children. Findings in 13 cases. Mayo Clin Proc 47:318-326, 1972

23. McNicol AM, Teasdale GM, Beastall GH: A study of corticotroph adenomas in Cushing's disease: no evidence of intermediate lobe origin. Clin Endocrinol (Oxf) 24:715-722, 1986

24. Melmed S, Casanueva FF, Hoffman AR, Kleinberg DL, Montori VM, Schlechte JA, et al: Diagnosis and treatment of hyperprolactinemia: an Endocrine Society clinical practice guideline. J Clin Endocrinol Metab 96:273-288, 2011

25. Mindermann T, Wilson CB: Pediatric pituitary adenomas. Neurosurgery 36:259-269, 1995

26. Molitch ME: Management of prolactinomas during pregnancy. J Reprod Med 44 (12 Suppl):1121-1126, 1999

27. Molitch ME, Cowen L, Stadiem R, Uihlein A, Naidich M, Russell E: Tumors invading the cavernous sinus that cause internal carotid artery compression are rarely pituitary adenomas. Pituitary 15:598-600, 2012

28. Nakane T, Kuwayama A, Watanabe M, Takahashi T, Kato T, Ichihara K, et al: Long term results of transsphenoidal adenomectomy in patients with Cushing's disease. Neurosurgery 21:218-222, 1987

29. Partington MD, Davis DH, Laws ER Jr, Scheithauer BW: Pituitary adenomas in childhood and adolescence. Results of transsphenoidal surgery. J Neurosurg 80:209-216, 1994

30. Patil CG, Prevedello DM, Lad SP, Vance ML, Thorner MO, Katznelson L, et al: Late recurrences of Cushing's disease after initial successful transsphenoidal surgery. J Clin Endocrinol Metab 93:358-362, 2008 
31. Perry A, Graffeo CS, Marcellino C, Pollock BE, Wetjen NM, Meyer FB: Pediatric pituitary adenoma: case series, review of the literature, and a skull base treatment paradigm. J Neurol Surg B Skull Base 79:91-114, 2018

32. Pollack IF: Brain tumors in children. N Engl J Med 331:1500-1507, 1994

33. Raffel C, Boggan JE, Eng LF, Davis RL, Wilson CB: Pituitary adenomas in Cushing's disease: do they arise from the intermediate lobe? Surg Neurol 30:125-130, 1988

34. Rey-Dios R, Payner TD, Cohen-Gadol AA: Pituitary macroadenoma causing symptomatic internal carotid artery compression: surgical treatment through transsphenoidal tumor resection. J Clin Neurosci 21:541-546, 2014

35. Ritzén EM, Wettrell G, Davies G, Grant DB: Management of pituitary gigantism. The role of bromocriptine and radiotherapy. Acta Paediatr Scand 74:807-814, 1985

36. Rollin GA, Ferreira NP, Junges M, Gross JL, Czepielewski MA: Dynamics of serum cortisol levels after transsphenoidal surgery in a cohort of patients with Cushing's disease. J Clin Endocrinol Metab 89:1131-1139, 2004

37. Rostomyan L, Daly AF, Petrossians P, Nachev E, Lila AR, Lecoq AL, et al: Clinical and genetic characterization of pituitary gigantism: an international collaborative study in 208 patients. Endocr Relat Cancer 22:745-757, 2015

38. Sedgh G, Finer LB, Bankole A, Eilers MA, Singh S: Adolescent pregnancy, birth, and abortion rates across countries: levels and recent trends. J Adolesc Health 56:223-230, 2015

39. Spallone A: Occlusion of the internal carotid artery by intracranial tumors. Surg Neurol 15:51-57, 1981

40. Tyrrell JB, Brooks RM, Fitzgerald PA, Cofoid PB, Forsham $\mathrm{PH}$, Wilson CB: Cushing's disease. Selective trans-sphenoidal resection of pituitary microadenomas. N Engl J Med 298:753-758, 1978

41. Wilson CB: Role of surgery in the management of pituitary tumors. Neurosurg Clin N Am 1:139-159, 1990

42. Wilson CB, Tyrrell JB, Fitzgerald PA, Forsham PH: Cushing's disease: surgical management, in Givens JR (ed): Hormone-Secreting Pituitary Tumors. Chicago: Year Book Medical Publishers, 1982, pp 199-208

43. Yaghmai R, Olan WJ, O'Malley S, Bank WO: Nonhemorrhagic pituitary macroadenoma producing reversible internal carotid artery occlusion: case report. Neurosurgery 38:1245-1248, 1996
44. Yao Y, Liu Y, Wang L, Deng K, Yang H, Lu L, et al: Clinical characteristics and management of growth hormone excess in patients with McCune-Albright syndrome. Eur J Endocrinol 176:295-303, 2017

45. Zadik Z, Cooper M, Chen M, Stern N: Cushing's disease presenting as pubertal arrest. J Pediatr Endocrinol Metab 6:201-204, 1993

46. Zimmerman D, Young WF Jr, Ebersold MJ, Scheithauer BW, Kovacs K, Horvath E, et al: Congenital gigantism due to growth hormone-releasing hormone excess and pituitary hyperplasia with adenomatous transformation. J Clin Endocrinol Metab 76:216-222, 1993

\section{Disclosures}

The authors report no conflict of interest concerning the materials or methods used in this study or the findings specified in this paper.

\section{Author Contributions}

Acquisition of data: Perry. Drafting the article: J Meyer, Oushy. Critically revising the article: Oushy, Graffeo. Reviewed submitted version of manuscript: FB Meyer, Oushy, Graffeo, Carlstrom. Study supervision: FB Meyer.

\section{Supplemental Information}

Previous Presentations

Components of this study were presented at the North American Skull Base Society 28th Annual Meeting held in Coronado, California, on February 16-18, 2018.

\section{Correspondence}

Fredric B. Meyer: Mayo Clinic, Rochester, MN. meyer.fredric@ mayo.edu. 